\title{
Disruption of a rice gene for $\alpha$-glucan water dikinase, OsGWD1, leads to hyperaccumulation of starch in leaves but exhibits limited effects on growth
}

\section{Tatsuro Hirose ${ }^{1,2 * t}$, Naohiro Aoki ${ }^{2 \dagger}$, Yusuke Harada ${ }^{2}$, Masaki Okamura ${ }^{2}$, Yoichi Hashida ${ }^{2}$, Ryu Ohsugi $^{2}$, Akio Miyao ${ }^{3}$, Hirohiko Hirochika ${ }^{3}$ and Tomio Terao ${ }^{1}$}

' NARO Agricultural Research Center, Niigata, Japan

${ }^{2}$ Graduate School of Agricultural and Life Sciences, The University of Tokyo, Tokyo, Japan

${ }^{3}$ National Institute for Agrobiological Sciences, Ibaraki, Japan

\section{Edited by:}

Thomas L. Slewinski, Cornell

University, USA

\section{Reviewed by:}

Alison Smith, John Innes Centre, UK L. C. Hannah, University of Florida, USA

\section{*Correspondence:}

Tatsuro Hirose, NARO Agricultural Research Center, 1-2-1 Inada, Joetsu, Niigata 943-0193, Japan.

e-mail:dragon@affrc.go.jp

${ }^{\dagger}$ Tatsuro Hirose and Naohiro Aoki have contributed equally to this work and should be regarded as joint first authors.
To identify potential regulators of photoassimilate partitioning, we screened for rice mutant plants that accumulate high levels of starch in the leaf blades, and a mutant line leaf starch excess 1 (LSE1) was obtained and characterized. The starch content in the leaf blades of LSE1 was more than 10-fold higher than that in wild-type plants throughout the day, while the sucrose content was unaffected. The gene responsible for the LSE1 phenotype was identified by gene mapping to be a gene encoding $\alpha$-glucan water dikinase, OsGWD1 (Os06g0498400), and a 3.4-kb deletion of the gene was found in the mutant plant. Despite the hyperaccumulation of starch in their leaf blades, LSE1 plants exhibited no significant change in vegetative growth, presenting a clear contrast to the reported mutants of Arabidopsis thaliana and Lotus japonicus in which disruption of the genes for $\alpha$-glucan water dikinase leads to marked inhibition of vegetative growth. In reproductive growth, however, LSE1 exhibited fewer panicles per plant, lower percentage of ripened grains and smaller grains; consequently, the grain yield was lower in LSE1 plants than in wild-type plants by $20 \sim 40 \%$. Collectively, although $\alpha$-glucan water dikinase was suggested to have universal importance in leaf starch degradation in higher plants, the physiological priority of leaf starch in photoassimilate allocation may vary among plant species.

Keywords: assimilate partitioning, gene disruption mutant, $\alpha$-glucan water dikinase, leaf starch, rice

\section{INTRODUCTION}

Assimilate partitioning has long been recognized as a target for crop improvement because it can limit the yield potential of the crop plants. Despite the great importance of rice as a major crop, most of the mechanisms of photoassimilate allocation in rice are yet to be elucidated. Utilizing mutants that exhibit phenotypes relevant to a particular biological phenomenon can be a powerful approach to uncovering potential regulators of that phenomenon. In the source leaves of higher plants, both starch and sucrose are the major primary products of photosynthesis; therefore any anomaly in the accumulation of the two compounds may arise from mutations in the genes involved in assimilate allocation. In particular, the accumulation of an extraordinarily high level of leaf starch is easily recognizable by iodine staining, and thus has been repeatedly reported as the "starch excess" phenotype. Starch excess in the leaves is often observed when any inhibition occurs (1) in the export of photoassimilate from source leaves or (2) in starch metabolism in the leaves. The former case includes disruption or suppression of the phloem-loading sucrose transporter (Bürkle et al., 1998; Gottwald et al., 2000) and blockade of the route of assimilate transport (Russin et al., 1996). Cold-girdling of the stem is also known to induce the starch excess phenotype through the impairment of photoassimilate transport (e.g., Krapp et al.,
1993). As an instance of the latter case, the Arabidopsis thaliana Starch EXcess 1 (SEX1) mutant (Caspar et al., 1991) is the most well-known because of its significant contribution to the current understanding of leaf starch metabolism, i.e., $\alpha$-glucan water dikinase (GWD, EC 2.7.9.4)-mediated degradation. Disruption of the plastid-localized maltose translocator (Niittylä et al., 2004) is also known to result in starch excess in the leaf, as well as some other mutations of starch degradation-related enzymes (Critchley et al., 2001). As is clear from the above described examples, the study of mutant plants exhibiting the starch excess phenotype in their leaves is a good approach to acquiring a better understanding of the mechanisms and regulation of photoassimilate allocation.

Thus far, no rice mutant that accumulates a high level of starch in the leaf blades has been reported. However, in our preliminary experiments, we found that pronounced starch accumulation occurred in detached leaf blades when sucrose was fed through their cut ends. This finding suggested that a rice mutant exhibiting the starch excess phenotype could exist and that it might be caused by a disorder in the mechanism controlling photoassimilate partitioning. We therefore decided to screen for mutant lines accumulating high levels of starch in their leaf blades. Here we report the isolation and characterization of the first leaf starch excess mutant of rice. 


\section{MATERIALS AND METHODS SCREENING OF THE LEAF STARCH EXCESS MUTANTS}

In this study, we used a collection of rice mutant lines induced by the insertion of the endogenous retrotransposon Tos17 (Miyao et al., 2003). To screen for mutants displaying the leaf starch excess (LSE) phenotype, 20 seeds of the M2 or M3 generation of Tos17 mutant lines were grown to the fourth leaf stage in the greenhouse. The terrestrial parts were sampled in the morning and leaf starch was visualized by iodine staining.

\section{GENE MAPPING}

Mapping populations were obtained from crosses between mutants with the japonica background and an indica/japonica crossbred rice cultivar "Takanari." In the F2 generation, mutant plants were selected by iodine staining of the terrestrial parts. DNA was extracted according to the method reported by Wang et al. (1993) from the leaf segments of plants showing the mutant phenotype. Gene mapping was carried out using micro-satellite SSR markers (McCouch et al., 2002).

\section{ANALYSIS OF THE GENE STRUCTURE}

Genomic DNA was extracted from green leaves by using the DNeasy Plant Kit (Qiagen, Valencia, CA, USA) and was used as the template for polymerase chain reaction (PCR) with PrimeStar GXL DNA Polymerase (Takara Bio Inc., Shiga, Japan) following the manufacturer's instructions. The nucleotide sequences of the PCR primers are listed in Table 1. For the analysis of the transcript structures, total RNA was extracted from the leaf blades and reverse-transcribed as below, and the resultant cDNA was used as the template for PCR with ExTaq DNA polymerase (Takara Bio Inc.).

\section{GENERATION OF TRANSGENIC RICE PLANTS}

For complementation analysis a 13.2-kb genomic DNA fragment containing the entire open reading frame (ORF) along with 2.2$\mathrm{kb}$ sequence upstream of the putative translation start point of OsGWD1 (Os06g0498400) was amplified by PCR using the primer

Table 1 | List of the PCR primers used in this study.

\begin{tabular}{|c|c|c|c|}
\hline Gene & Primer & Nucleotide sequence & Purpose* \\
\hline \multirow[t]{9}{*}{ OsGWD1 } & PL2 & ttgccttctgttcgccttaaaa & C \\
\hline & $\llcorner 4$ & ctctcccaaggtactgggt & I, S \\
\hline & L17 & tgaagccacgtgagataagc & S \\
\hline & L18 & gcttaaagggatggaatcaagc & S \\
\hline & L19 & gcagaagctggccaggcagt & $\mathrm{R}$ \\
\hline & $\mathrm{R} 2$ & gttatacatgtcccacggc & 1 \\
\hline & $\mathrm{R} 14$ & aacattgcctgattgacttggcta & $\mathrm{C}, \mathrm{S}$ \\
\hline & $\mathrm{R} 23$ & ctgccaacttccaagctg & S \\
\hline & $\mathrm{R} 24$ & acgttgcgagacttggcccc & $\mathrm{R}$ \\
\hline \multirow[t]{2}{*}{ RUBIO1 } & L1 & ggagctgctgctgttcttgg & $\mathrm{R}$ \\
\hline & $\mathrm{R} 1$ & cacaatgaaacgggacacga & $\mathrm{R}$ \\
\hline
\end{tabular}

${ }^{*} C$, gene construct for functional complementation; I, gene construct for RNA suppression; $R$, real-time RT-PCR; $S$, analysis of the transcript structure. pair, GWD1-PL2/R14 (Table 1), genomic DNA of the rice cultivar Nipponbare (as the template), and PrimeStar GXL DNA polymerase (Takara Bio Inc.). The DNA fragment was introduced into a transformation vector, pZH2B (Kuroda et al., 2010). For gene suppression analysis, a $0.4-\mathrm{kb}$ fragment of OsGWD1 was amplified using ExTaq DNA polymerase (Takara Bio Inc.), with the GWD1L4/R2 primer pair (Table 1) and cDNA from green seedlings, and introduced into the RNAi transformation vector, pANDA (Miki and Shimamoto, 2004). Transgenic rice plants were generated using the Agrobacterium tumefaciens-mediated method described by Hiei etal. (1994), and grown in a temperature-controlled glasshouse $\left(30 / 23^{\circ} \mathrm{C}\right.$, day/night $)$.

\section{QUANTITATIVE RT-PCR ANALYSIS}

For the analyses of mRNA abundance, rice plants were grown in plastic pots filled with nursery soil for rice seedlings. For tissuespecific expression analysis, rice seedlings were grown in a greenhouse under natural light conditions. For expression analyses of diurnal changes, rice plants were grown in a growth chamber with a 14 -h light $\left(27^{\circ} \mathrm{C}\right) / 10$-h dark $\left(23^{\circ} \mathrm{C}\right)$ photoperiod. The light intensity in the light period was $350 \mu \mathrm{mol} \mathrm{m} \mathrm{m}^{-2} \mathrm{~s}^{-1}$ and the light was emitted by fluorescent light tubes. Once the tissue samples were obtained from the plants, they were immediately frozen in liquid nitrogen and stored at $-80^{\circ} \mathrm{C}$ until use. Total RNA was extracted from various tissue samples using the extraction buffer described by Chang et al. (1993), although polyvinylpyrrolidone and spermidine were excluded. The extracted RNA was then purified using an RNeasy Mini Kit (Qiagen) followed by a TURBO DNA-free Kit (Ambion, Austin, TX, USA) to remove DNA. The purified RNA was reverse-transcribed and then an aliquot of the first-strand cDNA mixtures corresponding to $5 \mathrm{ng}$ of total RNA was used as the template for real-time quantitative reverse transcription (RT)PCR analysis using the SuperScriptIII Platinum Two-Step qRTPCR Kit with SYBR Green (Life Technologies, Austin, TX, USA). The reaction was carried out using an ABI7300 system (Applied Biosystems, Foster City, CA, USA) with the gene-specific primers listed in Table 1. In preliminary experiments, aliquots of the PCR reactions were electrophoretically separated on agarose gels to verify the specificity of the primers. The specificity of each PCR amplification was also checked using a heat dissociation protocol with temperatures changing from 60 to $95^{\circ} \mathrm{C}$ following the final cycle of the PCR. The results obtained for each CDNA were standardized to the expression of the rice polyubiquitin gene (RUBIQ1), which is a constitutively expressed gene in rice (Wang et al., 2000).

\section{DETERMINATION OF STARCH AND SOLUBLE SUGAR CONTENTS}

Frozen leaf samples were ground using a mortar and pestle under cryogenic conditions. A portion of the ground tissue $(\sim 25 \mathrm{mg}$ per sample) was immediately weighed and then extracted twice with $80 \%$ ethanol at $80^{\circ} \mathrm{C}$. After centrifugation at $8500 \mathrm{~g}$ for $5 \mathrm{~min}$, the supernatant was dried in vacuo, dissolved in distilled water, and used to assay sucrose, glucose, and fructose by the enzymatic method using F-kit \#716260 (J. K. International, Tokyo, Japan), which contains an invertase, a hexokinase, a glucose 6-phosphate dehydrogenase (G6PDH), and a phosphoglucomutase. The residual pellet obtained after centrifugation was dried in air, resuspended in distilled water, gelatinized by boiling for 
$4 \mathrm{~h}$, and used to assay starch by the enzymatic method using F-kit \#207748 (J. K. International), which contains an amyloglucosidase, a hexokinase, and a G6PDH. The assays were conducted according to the manufacturer's instructions, and the increase in the absorbance at $340 \mathrm{~nm}$ in each assay, resulting from the enzymatic conversions of starch, sucrose, glucose, or fructose into 6-phosphogluconate, were measured with a 96-well plate reader (Viento XS; Dainippon Sumitomo Pharma, Osaka, Japan).

\section{DETERMINATION OF THE PHOSPHORYLATION STATUS OF STARCH}

Fully elongated leaf blades of 3-week-old seedlings, grown under natural light conditions, were sampled $2 \mathrm{~h}$ after sunset, and frozen immediately. Approximately $200 \mathrm{mg}$ of frozen leaves were used in order to extract the starch, using the method as described above with slight modifications. The pellet after centrifugation was washed with distilled water three times to remove soluble metabolites completely, and resuspended in $0.5 \mathrm{~mL}$ distilled water. The resuspended pellet was gelatinized by boiling for $4 \mathrm{~h}$; then, it was digested thoroughly to hexose units by adding $0.1 \mathrm{~mL}$ amyloglucosidase (TOYOBO, Tokyo, Japan) at a concentration of $50 \mathrm{U} \mathrm{mL}^{-1}$ in $50 \mathrm{mM}$ sodium acetate buffer $(\mathrm{pH} 4.6)$ and incubating the solution at $60^{\circ} \mathrm{C}$ for $18 \mathrm{~h}$ with gentle shaking. Using the resultant digested starch samples, we first measured the amount of glucose, $[\mathrm{Glc}]_{\text {starch }}$, by the same enzymatic method as described above. Next, as for the same digested starch samples, we also measured the amount of glucose 6-phosphate, $[\mathrm{G} 6 \mathrm{P}]_{\text {starch }}$, by another enzymatic method using highly purified G6PDH (Roche Diagnostics, Mannheim, Germany) as the sole enzyme, to allow the conversion of only G6P to 6-phosphogluconate. More than $30 \mu \mathrm{g}$ of starch (equivalent to $185 \mathrm{nmol}$ of the hexose unit) was used to assay G6P in a $200-\mu \mathrm{L}$ reaction, in order to obtain significant increases in the absorbance at $340 \mathrm{~nm}$. No significant changes in the absorbance were detected when $200 \mathrm{nmol}$ of authentic glucose instead of digested starch samples were added into the $200-\mu \mathrm{L}$ assay mixture, indicating that the hexokinase activity contamination from the G6PDH enzyme solution was negligible.

Since the enzymatic assay of [Glc $]_{\text {starch }}$ provides the total concentration of Glc plus G6P, the phosphorylation status of starch was calculated according to the following equation:

$[\mathrm{G} 6 \mathrm{P}]_{\text {starch }} /[\mathrm{Glc}]_{\text {starch }} \times 100$

$=$ percentage of phosphorylated glucose residue in starch $(\%)$.

\section{MEASUREMENTS OF PHOTOSYNTHESIS RATE AND ${ }^{13}$ C-PHOTOASSIMILATE PARTITIONING}

Rice plants were grown in plastic pots in glasshouses until the 6 th to 7 th leaf stage and then used for the experiments. Net $\mathrm{CO}_{2}$-assimilation rates in the leaf blades were measured using a portable photosynthesis system (LI-6400, LI-COR, Lincoln, NE, USA). Analysis of ${ }^{13} \mathrm{C}$-photoassimilate partitioning in rice plants was conducted according to the method described by Sasaki et al. (2005) with modifications. In brief, ${ }^{13} \mathrm{CO}_{2}$ was fed to rice seedlings in sealed, transparent plastic box, for $2 \mathrm{~h}$ (from 11:00 to 13:00) under the light intensity of around $750 \mu \mathrm{mol} \mathrm{m}^{-2} \mathrm{~s}^{-1}$, in a controlled glasshouse $\left(27 / 23^{\circ} \mathrm{C}, 14\right.$-h day/10-h night, the day period from 5:00 to 19:00). ${ }^{13} \mathrm{CO}_{2}$ gas was liberated from $\mathrm{Ba}^{13} \mathrm{CO}_{3}$ pow$\operatorname{der}(\geq 98$ atom $\%$, Cambridge Isotope Laboratories, Andover, MA,
USA) mixed with $7.3 \mathrm{M} \mathrm{H}_{3} \mathrm{PO}_{4}$ inside the box. After the ${ }^{13} \mathrm{C}$ feeding was ceased by removing the plastic box, the aboveground parts of the seedlings were sampled at designated times; immediately after the cessation of ${ }^{13} \mathrm{C}$-feeding (13:00), in the evening of the same day (19:00), and in the morning of the next day (7:00). The aboveground samples were divided into leaf blades and sheaths, and then stored at $-80^{\circ} \mathrm{C}$ until use. For measuring ${ }^{13} \mathrm{C}$ contents, the frozen tissue samples were freeze-dried, followed by measuring dry weight. The dried tissue materials were ground to a fine powder, and a portion (ca. $200 \mu \mathrm{g}$ ) of the tissue powder was used for the determination of ${ }^{13} \mathrm{C}$ content. The total carbon and ${ }^{13} \mathrm{C}$ contents were determined using an elemental analyzer (NC2500, Thermoquest, San Jose, CA, USA) and a mass spectrometer (Delta Plus System, Thermoquest). The ${ }^{13} \mathrm{C}$ content in each tissue sample was calculated according to the equation of Sasaki et al. (2005). Partitioning of ${ }^{13} \mathrm{C}$-photoassimilates to the leaf blades was calculated from the total amounts of ${ }^{13} \mathrm{C}$ in leaf blades, $\left[{ }^{13} \mathrm{C}\right]_{\mathrm{LB}}$, and sheaths, $\left[{ }^{13} \mathrm{C}\right]_{\mathrm{LS}}$, according to the following equation:

$$
\begin{aligned}
& {\left[{ }^{13} \mathrm{C}\right]_{\mathrm{LB}} /\left\{\left[{ }^{13} \mathrm{C}\right]_{\mathrm{LB}}+\left[{ }^{13} \mathrm{C}\right]_{\mathrm{LS}}\right\} \times 100} \\
& \quad={ }^{13} \mathrm{C} \text {-partitioning to leaf blades }(\%) .
\end{aligned}
$$

\section{FIELD TRIALS AND DETERMINATION OF YIELD COMPONENTS AND GRAIN YIELD}

Field trials were carried out in paddy fields at the Institute for Sustainable Agro-ecosystem Services (ISAS), Tokyo, Japan $\left(35^{\circ} 44^{\prime} \mathrm{N}\right.$, $139^{\circ} 32^{\prime} \mathrm{E}$ ). Seeds were sown in a greenhouse in late April, and transplanted into the paddy fields in late May. In any given year, the planting density was 22.2 hills per square meter (hill spacing of $30 \mathrm{~cm} \times 15 \mathrm{~cm}$ ) with one seedling per hill, and compound fertilizer for paddy fields $\left(\mathrm{N}: \mathrm{P}_{2} \mathrm{O}_{5}: \mathrm{K}_{2} \mathrm{O}=12: 16: 18 \%\right)$ was applied at the rate of $50 \mathrm{~g} \mathrm{~m}^{-2}$ as a basal dressing. Approximately 45 days after the heading, plants were harvested in late September, and their panicles were used for the analysis of yield components. The panicle number per hill (A) and spikelet number per panicle (B) were counted by hands. Subsequently, ripened grains were selected as unhulled grains that settle down in a salt solution with a density of $1.06 \mathrm{~g} \mathrm{~mL}^{-1}$. The percentage of ripened grains (C) was calculated from the total spikelet number per hill and the ripened grain number. Unhulled grain yield was calculated from the yield components measured and the planting density according to the following equation.

$$
\begin{gathered}
\mathrm{A} \times \mathrm{B} \times \mathrm{C} / 100 \times[\text { grain weight }(\mathrm{g} 1000 \text {-grain } \\
\end{gathered}
$$

\section{RESULTS}

\section{SCREENING OF THE LEAF STARCH EXCESS MUTANTS AND THE LSE1 PHENOTYPE}

Of 6377 Tos 17 mutant lines, 5 were selected by iodine staining to detect distinct accumulation of leaf starch (stain-positive plants). One of the five lines designated as ND0717, exhibited dark staining for starch in the entire terrestrial portion while the wild-type plants did not show any discernible staining (Figure 1). Because the seeds originally used for the screening were M2 seeds, i.e., those borne 


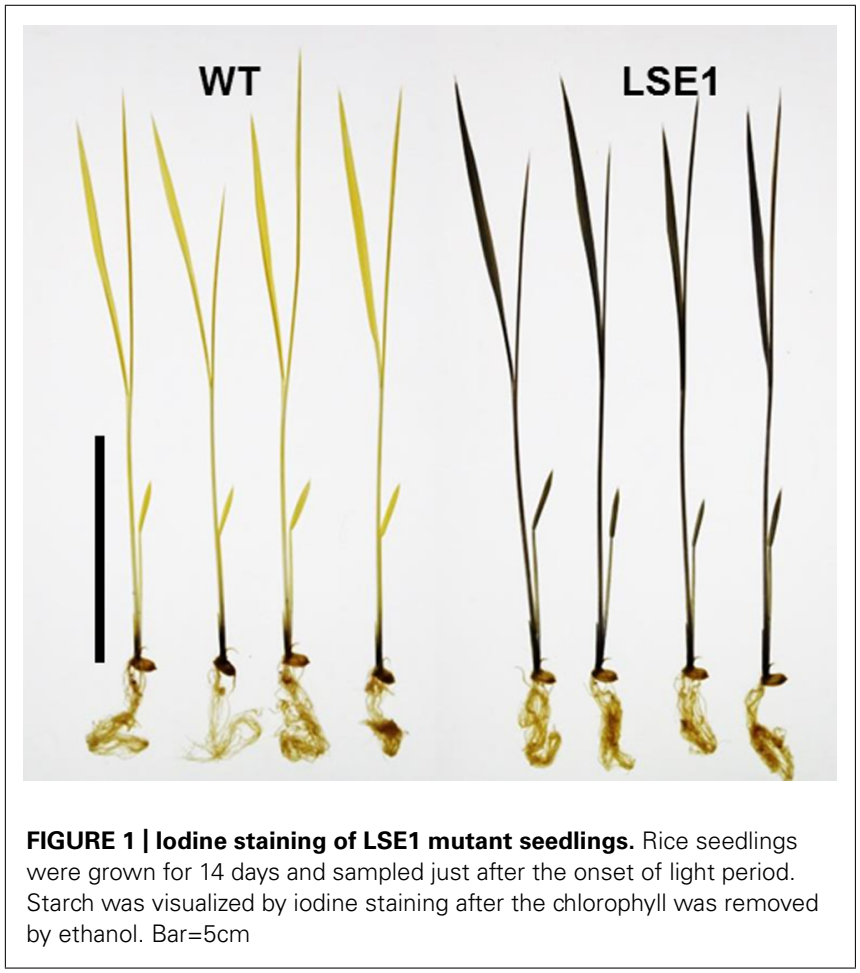

on the M1 generation plants, they segregated into stain-positive and stain-negative plants. The progeny of these M2 plants were further tested for leaf starch accumulation, and in the M3 generation, the progeny of some stain-negative M2 plants segregated into stain-positive and stain-negative ones at a ratio of 1:3, suggesting that the LSE phenotype was due to the recessive mutation of a single gene. To establish homozygously mutated lines, the progeny of stain-positive M2 plants were examined to check whether all the individuals showed the stain-positive phenotype. Based on the results of this analysis, of the 15 original M2 plants, 2 were identified as having the homozygous mutant genotype, 2 as wildtype, and the remaining 11 as having the heterozygous genotype. After this, we used the progeny of the two putative homozygous mutant plants as the pure line for the mutation and designated this mutation LSE1, while the progeny of the two putative wild-type genotype plants were used as controls in the various assays in this study and were designated "wild-type." The starch content in the mature leaf blades of LSE1 plants was 5- to 10-fold higher than that in the wild-type plants throughout the day (Figure 2A). Contrastingly, the sucrose content in the leaf blades was not different between the two (Figure 2B).

\section{GENE MAPPING OF THE LSE1 MUTATION}

Southern blot analysis revealed that the LSE phenotype of LSE1 was not tagged by the Tos 17 retrotransposon; it was therefore believed to be caused by some other mutation (data not shown). In order to identify the responsible gene for the mutation, gene mapping was carried out. The responsible gene was mapped to a $1.16-\mathrm{Mb}$ region on chromosome 6 flanked by two genetic markers RM20061 and RM20104 (McCouch et al., 2002). Although as many as 77 genes are annotated within this region, it was significant that a gene for a putative GWD, Os06g0498400, was located in this region because disruption of the corresponding orthologs of this gene results in the LSE phenotype in both Arabidopsis (SEX1 mutant; Caspar et al., 1991) and Lotus japonicus (Vriet et al., 2010). Therefore, we decided to further explore this gene as a major candidate gene causing the LSE1 phenotype, and the gene Os06g0498400 was designated as OsGWD1. As the first

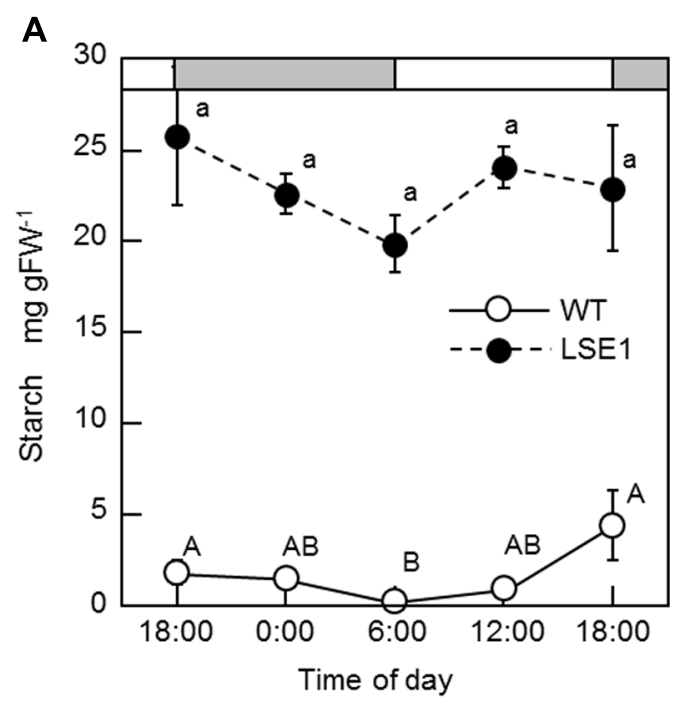

FIGURE 2 | Diurnal changes in starch (A) and sucrose (B) levels. Rice seedlings were grown in a growth chamber with a 12-h light (6:00-18:00)/12-h dark photoperiod. Temperature was maintained at $25^{\circ} \mathrm{C}$ during the light period and at $20^{\circ} \mathrm{C}$ during the dark period. White and gray bars represent light and dark periods, respectively. Fully expanded leaf blades of 3-week-old seedlings

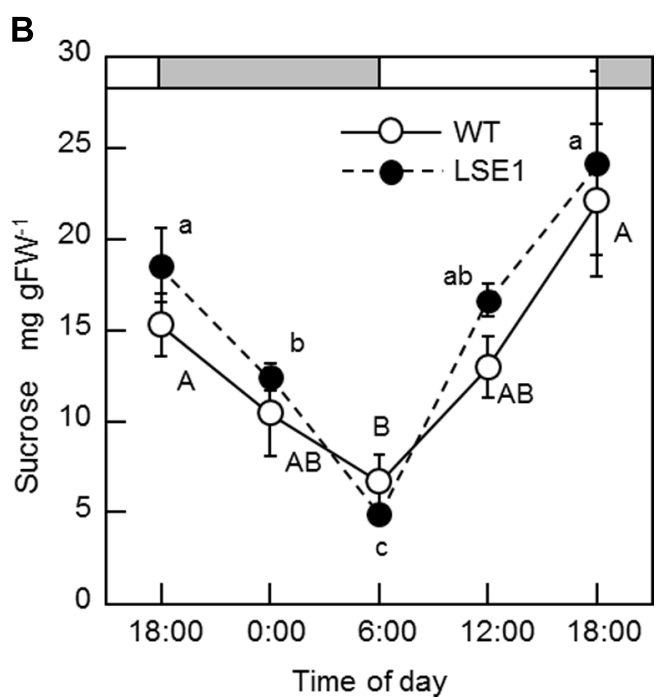

were harvested at designated times of the day and used for the measurements. Data represent the mean \pm SE $(n=5)$ and the different letters indicate significant differences within each of the two lines, capital letters for WT and small letters for LSE1 $(p<0.05$, Tukey's test). 
step, we examined whether the structure of the OsGWD1 gene was different between the LSE1 mutant and the original cultivar Nipponbare, by genomic PCR analysis using several primer combinations designed based on the published DNA sequence of Os06g0498400. OsGWD1 has been predicted to be a relatively complex gene structure comprising as many as 32 exons, and it was found that the OsGWD1 gene in the LSE1 mutant had a long deletion of $3434 \mathrm{bp}$ corresponding to the nucleotide position from 7415 to 10758 of the reference sequence in the database, NC_008399. As a result, OsGWD1 in the LSE1 mutant lacks the exons from the 22nd to the 32nd (Figure 3A).

\section{EXPRESSION ANALYSIS OF OSGWD1 IN LSE1 AND THE WILD-TYPE PLANTS}

The transcript of OsGWD1 in the leaf blades was analyzed in both LSE1 and the original cultivar Nipponbare by RT-PCR. According to the gene structure and the sequences of the cDNA clones in the database, the transcript of OsGWD1 was predicted to be $\sim 4.4 \mathrm{~kb}$ in wild-type plants and to contain a 1421-bp deletion in the case of LSE1, and the primer pairs were designed to check the structure of the transcript (Figure 3B). With a primer pair that spanned the LSE1 deletion (L18/R14; Figure 3C), a shorter PCR product was amplified with the expected size in LSE1, and no PCR product was detected with a left primer that was located within the deletion (L4/R14; Figure 3C).
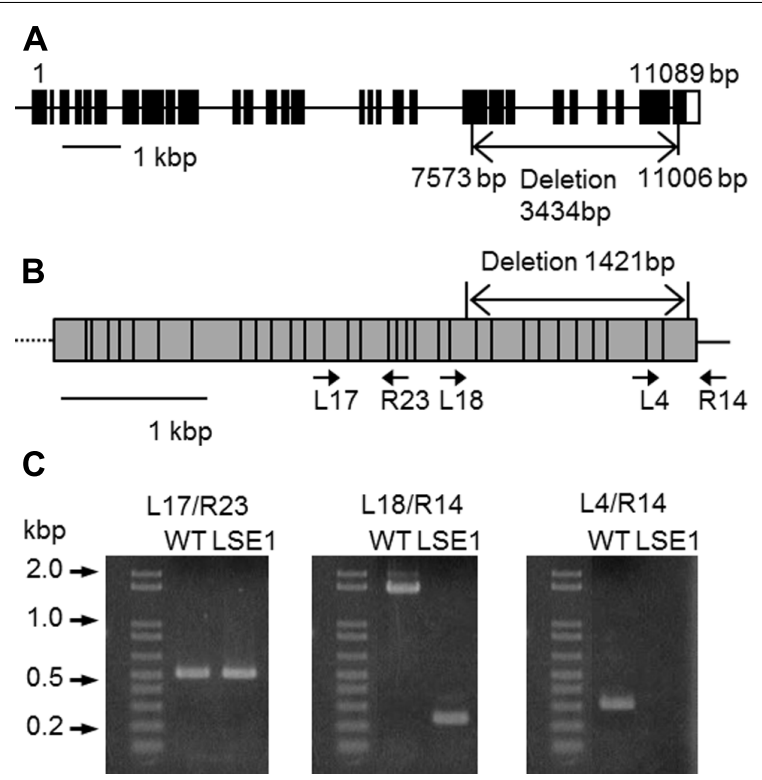

FIGURE 3 | Structure of OsGWD1 and its transcript. (A) The gene structure of OsGWD1 is illustrated; black boxes indicate exons and the position of the gene deletion in LSE1 mutant is shown by an arrow. (B) The coding region of the OSGWD1 transcript is indicated by a gray rectangle, in which the junctions of the exons are shown by vertical bars. The positions of the PCR primers used for the analysis of the transcript structure are indicated by the arrow heads (see below). Note that the primers L17, L18, L4, and R23 span the exon/intron junction on the cDNA. (C) Agarose gel electrophoresis images of the PCR products are presented to demonstrate that an aberrant transcript is accumulated in the leaves of LSE1 plants. The results of PCR with the different primer pairs are shown.
To determine the expression pattern of OsGWD1 in normal rice plants, real-time RT-PCR analysis was conducted using various tissues from 2-week-old plants and 4-day-old germinating seeds of Nipponbare. The mRNA levels in both leaf blades and sheaths were relatively high, while those in the roots were much lower (Figure 4A). The diurnal change in the mRNA level of OsGWD1 was also examined using the fully expanded leaf blades of 2-weekold plants, grown with a 14-h light period (Figure 4B). The mRNA level of OsGWD1 was highest in the early night (20:00) and then decreased until the next noon (12:00).

\section{FUNCTIONAL COMPLEMENTATION OF OsGWD1 IN THE LSE1 MUTANT}

To examine whether OsGWD1 is responsible for the LSE1 mutation, the mutant plants were introduced with a genomic DNA fragment from Nipponbare containing the entire ORF along with a 2.2-kb sequence upstream of the putative translation start point

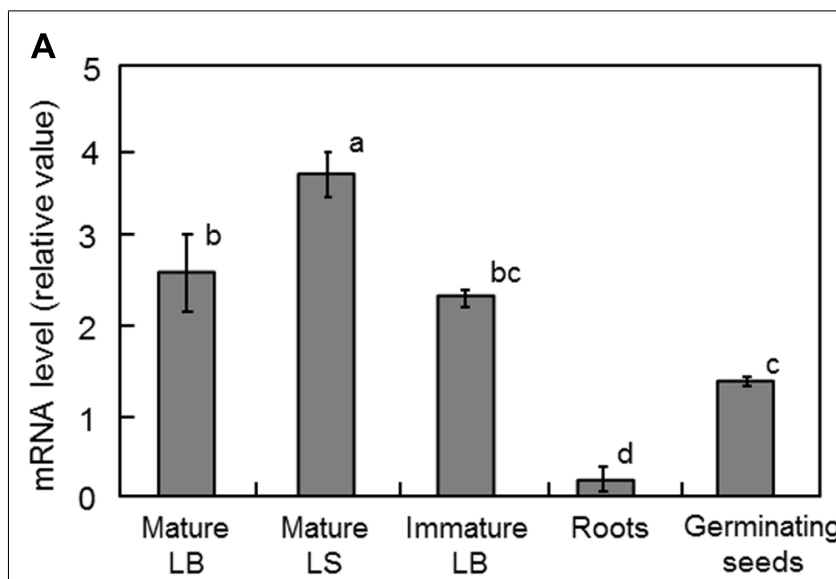

B

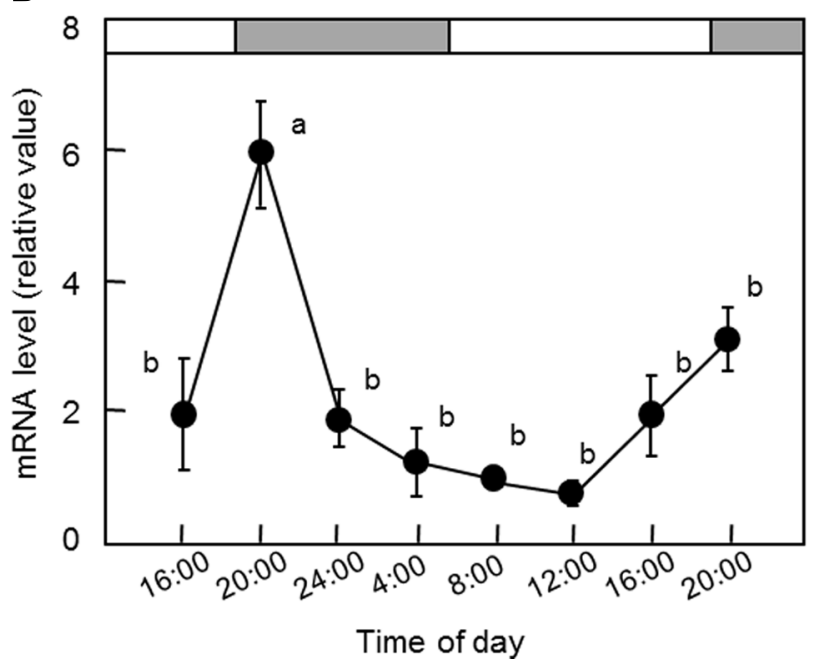

FIGURE 4 | Transcript levels of OsGWD1 in rice seedlings.

Tissue-specific accumulation pattern (A) and diurnal changes in leaf blades (B), with respect to the mRNA levels of OsGWD1 were examined by quantitative RT-PCR analysis. Values are standardized to the expression level of a rice polyubiquitin gene (\% RUBIQ1). Data represent the mean \pm SE $(n=4)$ and the different letters indicate significant differences ( $p<0.05$, Tukey's test). LB, leaf blade; LS, leaf sheath. 


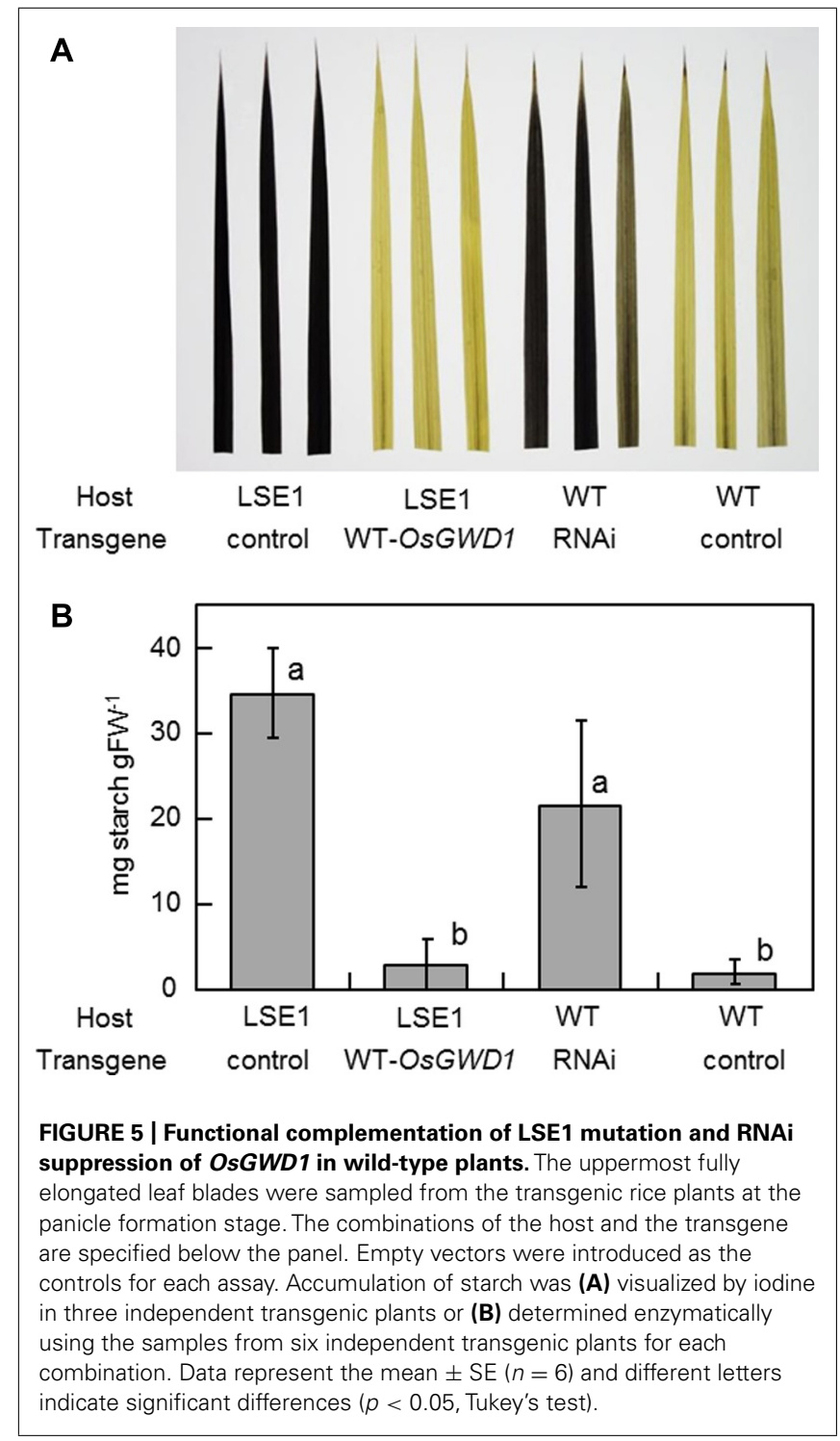

of OsGWD1. As expected, it was observed that OsGWD1 from Nipponbare restored the LSE1 phenotype to wild-type; the starch content in the leaf blades of the transgenic LSE1 plants was lower and was similar to that seen in the controls (Figure 5). In addition, when the RNAi construct for OsGWD1 was introduced into Nipponbare plants, the starch level in the leaf blades increased significantly compared to that in the controls, mimicking the phenotype of LSE1 (Figure 5).

\section{PHOSPHORYLATION STATUS OF STARCH IN LSE1 MUTANT}

Since GWD has been reported to play a role in the degradation pathway of starch, by catalyzing the phosphorylation of the C6 position of the glucosyl residue in amylose chains (see Blennow et al., 2002 for a review), we examined the extent of phosphorylation of the glucosyl residues in starch, by measuring the amount of G6P as well as that of glucose in digested starch extracted from mature leaf blades. Leaf samples were harvested $2 \mathrm{~h}$ after the sunset, during which the mobilization of leaf starch would occur actively.
In wild-type leaves, the percentage of phosphorylated glucosyl residues in the starch molecules was $0.20 \pm 0.04 \%$ (average \pm standard error, $n=4$ ), namely, 2 of every 1000 glucosyl residues were phosphorylated, which is comparable with the reported phosphorylation status of starch in Arabidopsis and potato plants (Yu et al., 2001; also see Blennow et al., 2002 for a review). In LSE1 leaves, the percentage of phosphorylation was $0.05 \pm 0.02 \%$, significantly lower than the wild-type leaves $(p<0.001$ by $t$-test, $n=4)$.

\section{VEGETATIVE GROWTH, LEAF PHOTOSYNTHESIS, AND ${ }^{13}$ C-PHOTOASSIMILATE PARTITIONING}

The vegetative growth of LSE1 was assessed by the leaf number on the main stem and the shoot dry weight. For both growth parameters, there was no significant difference between LSE1 and the wild-type control (Figure 6A). The rates of net photosynthesis in the leaf blades, measured under either ambient or saturating concentrations of $\mathrm{CO}_{2}$, were not significantly different between LSE1 and the wild-type (Figure 6B). The effect of the LSE1 mutation on the photoassimilate partitioning was accessed by ${ }^{13} \mathrm{C}$-tracer technique. Just after the cessation of the feeding $(0-\mathrm{h})$, approximately $70 \%$ of the ${ }^{13} \mathrm{C}$ remained in the leaf blades in both genotypes, and then it decreased gradually. The ${ }^{13} \mathrm{C}$-partitioning to the leaf blades at 6- and 18-h after feeding showed a tendency to be greater in LSE1 than in the wild-type, but the differences were not significant (Figure 6C).

\section{YIELD TRAITS OF LSE1}

To investigate the impact of the LSE1 mutation on grain productivity in rice plants, LSE1, wild-type, and the original cultivar Nipponbare were grown in paddy fields in Tokyo and Niigata, Japan, from 2009 to 2012. Essentially the same results were obtained in all the field trials, and Table 2 shows a representative result obtained in 2010 in Tokyo. Among the yield components of rice, the panicle number per hill, percentage of ripened grain, and 1000-grain weight were significantly lower in LSE1 plants than in wild-type and Nipponbare plants. As a result, grain yield reductions of $20 \sim 40 \%$ were observed in LSE1 compared with the control lines, in all the field trials conducted.

\section{DISCUSSION}

To date, a number of mutants have been reported for the hyperaccumulation of starch in their leaves. In maize, several mutant lines exhibiting the LSE phenotype have already been reported, for example, Sxd1 (Russin et al., 1996), Tdy1 (Braun et al., 2006), and Psc (Slewinski and Braun, 2010), all of which are related to photoassimilate export from the leaves. In rice, however, no such mutation has been documented so far; to our knowledge, LSE1 is the first such case. Gene mapping and gene structure analysis revealed that the LSE1 mutant has a deletion of as much as $3.4 \mathrm{~kb}$ in $O s G W D 1$, a putative gene for $\alpha$-glucan water dikinase, and consequently, accumulates aberrant mRNA lacking one-third of the coding region in the leaf blades (Figure 3 ). Therefore, LSE1 was assumed to be a loss-of-function mutant of OsGWD1. This hypothesis was strongly supported by the complementation test for the LSE1 mutation with an intact OsGWD1 and by RNAi-mediated suppression of OsGWD1 in Nipponbare plants (Figure 5). Disruption of the genes for GWD has already 

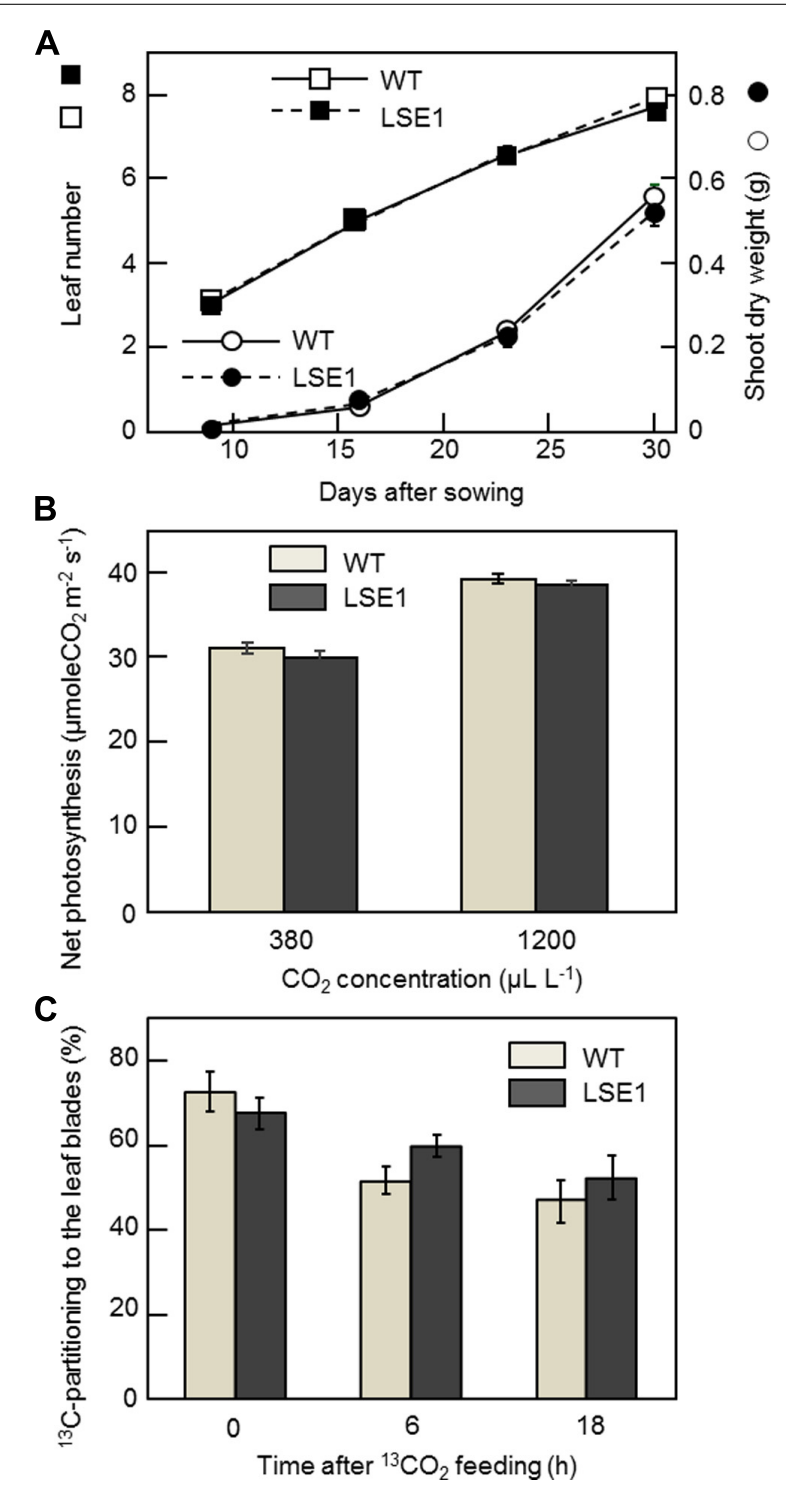

FIGURE 6 | Growth, photosynthesis and ${ }^{13} \mathrm{C}$-photoassimilate partitioning in the seedlings of LSE1. (A) The dry weight of the terrestrial portion (circles) and the leaf number (squares) were examined in the seedlings of LSE1 (closed symbols) and wild-type plants (open symbols) during the early vegetative growth stage. Data represent the mean $\pm \mathrm{SE}$ $(n=5)$. (B) Net photosynthesis rates of the uppermost fully elongated 7 th leaf blades were measured under $\mathrm{CO}_{2}$ concentrations of both 380 and $1200 \mu \mathrm{L} \mathrm{L}^{-1}$. Light intensity was set at $1200 \mu \mathrm{mol}$ photon $\mathrm{m}^{-2} \mathrm{~s}^{-1}$. Data represent the mean \pm SE $(n=4)$. (C) Partitioning of ${ }^{13} \mathrm{C}$-photoassimilate to the leaf blades was measured immediately $(0 \mathrm{~h}), 6 \mathrm{~h}$, and $18 \mathrm{~h}$ after the cessation of 2 -h feeding of ${ }^{13} \mathrm{CO}_{2}$. The seedlings at the 6 th leaf stage were used in the experiment. Data represent the mean $\pm \operatorname{SE}(n=4)$.

been reported to lead to the LSE phenotype in Arabidopsis (SEX1; Caspar et al., 1991), L. japonicus (Vriet et al., 2010), and tomato (Nashilevitz et al., 2009), all of which are observed to show LSE phenotype.

In normal plants, a higher level of the OsGWD1 transcript was observed in leaf blades and sheaths but a much lower level was seen in roots (Figure 4A), showing agreement with the pattern of starch accumulation in LSE plants visualized by iodine staining (Figure 1). The transcript level of OsGWD1 in leaf blades changed diurnally and peaked just after the onset of the dark period (Figure 4B), which seems reasonable as it is believed to play a role in the degradation of starch accumulated during the light period. A similar diurnal change in the GWD transcript level has been reported in Arabidopsis (Yu et al., 2001; Smith et al., 2004). However, GWD protein level was shown not to change diurnally (Yu et al., 2001; Lu et al., 2005). Further investigation including diurnal change in GWD activity is needed to better understand this problem. The biochemical and physiological functions of GWD have been extensively studied in the Arabidopsis SEX1 mutant and are now believed to involve phosphorylation of the glucosyl residues in starch, which facilitates amylolytic degradation (see Blennow et al., 2002 for a review). In the present study, it was observed that leaf starch from the LSE1 plants contained less G6P than that from wildtype plants, consistent with observations in Arabidopsis SEX1 (Yu et al., 2001). Accordingly, OsGWD1 appears to fulfill the same role in the process of leaf starch degradation in rice as in other plant species in which disruption mutants of GWD genes are reported. In other words, it was suggested that GWD has universal importance in the degradation of leaf starch among higher plants.

While LSE1 shares considerable similarity in its phenotype with the reported mutants of GWD, a conspicuous difference was found in the vegetative growth; LSE1 grows normally unlike the GWD mutants of Arabidopsis and L. japonicus (Figure 6A). The reason for this difference is not clear. However, it may be relevant to the physiological priority of leaf starch in the photoassimilates. In rice leaf blades, newly fixed carbon is preferentially directed to sucrose biosynthesis rather than starch, in contrast to other plant species such as Arabidopsis. This is evident from the fact that the content of starch and sucrose in rice leaf blades at the end of the day is ca. 5 and $20 \mathrm{mg}$ per gram flesh weight $\left(\mathrm{gFW}^{-1}\right)$, respectively, while that reported for Arabidopsis in the literatures is around 10 and $1 \mathrm{mg} \mathrm{gFW}^{-1}$, respectively (Figures 2A,B; e.g., Chia et al., 2004). Accordingly, rice may have sufficient capacity for the synthesis of sucrose to achieve normal growth without the transient starch in the chloroplast, and thus may experience limited impact on growth when the mobility of the transient starch is impaired by a lesion in OsGWD1. This view is reinforced by the observation that a knockout mutant of the ADP-glucose pyrophosphorylase (AGPase) gene, OsAPL1, grows normally although it accumulates less than 5\% of starch in the leaf blades in comparison with wild-type plants (Rösti et al., 2007). In addition, the sucrose synthesized in photosynthetic cells was considered to be exported from the source leaves of LSE1 as efficiently as wildtype, from the facts that (1) bulk concentration of sucrose in the leaf blades did not differ between the two genotypes throughout the day (Figure 2B), and (2) photoassimilate export estimated by decrease in the ${ }^{13} \mathrm{C}$-partitioning to the leaf blades after the tracer feeding was also comparable between the two (Figure 6C). Finally, the present results indicate that hyperaccumulation of starch in the source leaves does not inhibit photosynthesis in the leaves (Figure 6B), which has often been assumed in the research history of the regulation of photosynthesis (see Stitt, 1991 for a review). 
Table 2 | Yield components and grain yield of LSE1, wild-type and the original cultivar Nipponbare grown under field conditions.

\begin{tabular}{|c|c|c|c|c|c|}
\hline & $\begin{array}{l}\text { Panicle number } \\
\text { per plant }\end{array}$ & $\begin{array}{l}\text { Spikelet number } \\
\text { per panicle }\end{array}$ & $\begin{array}{l}\text { Percentage of } \\
\text { ripened grain }\end{array}$ & $\begin{array}{l}\text { Grain weight } \\
\text { (g } 1000 \text { grains }^{-1} \text { ) }\end{array}$ & Grain yield $\left(\mathrm{g} \mathrm{m}^{-2}\right)$ \\
\hline Wild-type & $9.5 \pm 0.2(100)$ & $105.8 \pm 3.0(100)$ & $74.8 \pm 1.6(100)$ & $26.5 \pm 0.3(100)$ & $444.1 \pm 8.9(100)$ \\
\hline LSE1 & $8.7 \pm 0.3^{*}(91)$ & $101.1 \pm 4.0(96)$ & $64.1 \pm 2.0^{* *}(86)$ & $25.1 \pm 0.2^{* *}(95)$ & $313.2 \pm 21.2^{* *}(71)$ \\
\hline Nipponbare & $9.0 \pm 0.3(95)$ & $115.8 \pm 4.8(109)$ & $78.9 \pm 0.8(105)$ & $26.7 \pm 0.1(101)$ & $488.9 \pm 31.0(110)$ \\
\hline
\end{tabular}

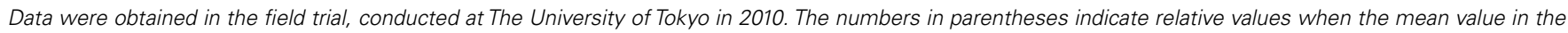

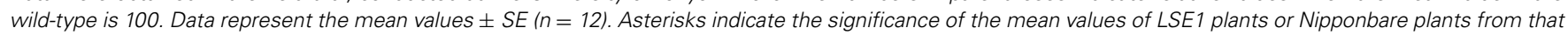

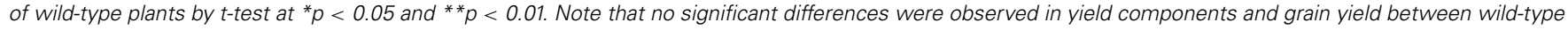
plants and the original cultivar Nipponbare, indicating that these two lines were similar with regard to growth and productivity in paddy fields.

Recently Weise et al. (2012) reported in maize that RNAi suppression of a GWD gene did not affect the vegetative growth in spite of a high level of starch accumulation in leaves. This, together with our results, suggests that vegetative growth of grass species is less dependent on GWD-mediated leaf starch remobilization than the dicot species reported.

Nevertheless, it should be also noted that the grain yield of LSE1 was significantly lower than that of wild-type plants and Nipponbare because of combined suppression of yield components (Table 2). This finding indicates that the impairment of GWD exerted some adverse effect on reproductive growth, although such effects are not apparent in vegetative growth. It is possible that the impairment of GWD in LSE1 slightly affected growth throughout the lifecycle in a manner that was not detectable in the short-term, and the significant reduction in the yield traits might be a manifestation of the cumulative effects. Information on the effect of GWD mutation on the reproductive growth is still limited, although Andriotis et al. (2012) recently reported that mature seeds of Arabidopsis SEX1 mutant have less lipid content than seeds of wild-type plants due to starvation of the carbohydrate in the fruit imported from the maternal plant. Here again, mutant plants lacking leaf starch can be a good reference because they share unavailability of leaf starch in common with LSE1. However, the results reported are inconsistent; AGPase mutants lacking leaf starch, rice apl1 (Rösti et al., 2007) and maize agps-m1 (Slewinski et al., 2008) showed no reduction in productivity under controlled environment whereas the latter mutant did exhibit yield reduction when grown in the field (Schlosser et al., 2012). It may be possible that the adverse effects of the mutation become apparent only in the field where plants routinely experience various environmental stresses unlike in the greenhouse. It may be also assumed that in LSE1 some disorder in starch metabolism in the stem reserve was brought about by the mutation because the stems of rice accumulate starch before heading to meet a large demand of carbohydrate for

\section{REFERENCES}

Andriotis, V. M. E., Pike, M. J., Schwarz, S. L., Rawsthorne, S., Wang, T. L., and Smith, A. M. (2012). Altered starch turnover in the maternal plant has major effects on Arabidopsis fruit growth and seed composition. Plant Physiol. 160, 1175-1186.
Blennow, A., Engelsen, S., Nielsen, T., Baunsgaard, L., and Mikkelsen, R. (2002). Starch phosphorylation: a new front line in starch research. Trends Plant Sci. 7, 445-450.

Braun, D. M., Ma, Y., Inada, N., Muszynski, M. G., and Baker, R. F. (2006). tie-dyed1 regulates

grain filling and also to buffer unfavorable environmental conditions after heading (see Slewinski, 2012 for a review). In our preliminary experiment, however, the stem starch content at the heading stage did not differ between LSE1 and the wild-type plants (data not shown), although the effects of the mutation on the mobilization of stem starch after heading are yet to be elucidated. A more extensive study with an agronomical perspective is clearly needed to uncover the mechanism of yield reduction in LSE1.

In conclusion, the LSE1 mutation in rice, i.e., disruption of OsGWD1, resulted in a mild phenotypic change at the whole plant level, highlighting the variable physiological priority of leaf starch among plant species. In addition to LSE1, we have isolated additional Tos17 mutant lines exhibiting the LSE phenotype, and some of these LSE mutants appear to exhibit a severe phenotype with respect to growth (Hirose, Aoki, Miyao, Hirochika, unpublished). Analysis of the LSE mutants as well as the identification of the responsible genes would increase our understandings of the molecular mechanism and/or physiological significance of photoassimilate allocation in rice.

\section{ACKNOWLEDGMENTS}

We would like to express our gratitude to Ms. Kiiko Takatsuto, NARO Agricultural Research Center for the excellent technical assistance; to Drs. Haruto Sasaki, Naoko Uehara, and Tomohito Egami for their help in the analysis of ${ }^{13} \mathrm{C}$-photoassimilate partitioning; and to the technical support staffs of the ISAS, The University of Tokyo for their cultivation management of rice. We measured ${ }^{13} \mathrm{C}$ by means of mass spectrometry at the Asia Natural Environmental Science Center, The University of Tokyo. This work was supported in part by the Japan Society for the Promotion of Science (JSPS), KAKENHI grant number 22380017, to Tatsuro Hirose, Naohiro Aoki, and Ryu Ohsugi. Masaki Okamura is a recipient of a fellowship from the JSPS.

carbohydrate accumulation in maize leaves. Plant Physiol. 142, 1511-1522.

Bürkle, L., Hibberd, J. M., Quick, W. P., Kühn, C., Hirner, B., and Frommer, W. B. (1998). The $\mathrm{H}+$-sucrose cotransporter NtSUT1 is essential for sugar export from tobacco leaves. Plant Physiol. 118, 59-68.

Caspar, T., Lin, T. P., Kakefuda, G., Benbow, L., Preiss, J., and Somerville, C. (1991). Mutants of Arabidopsis with altered regulation of starch degradation. Plant Physiol. 95, 1181-1188. 
Chang, S., Puryear, J., and Cairney, J. (1993). A simple and efficient method for isolating RNA from pine trees. Plant Mol. Biol. Rep. 11, 113-116.

Chia, T., Thorneycroft, D., Chapple, A., Messerli, G., Chen, J., Zeeman, S. C., et al. (2004). A cytosolic glucosyltransferase is required for conversion of starch to sucrose in Arabidopsis leaves at night. Plant J.37, 853-863.

Critchley, J. H., Zeeman, S. C., Takaha, T., Smith, A. M., and Smith, S. M. (2001). A critical role for disproportionating enzyme in starch breakdown is revealed by a knock-out mutation in Arabidopsis. Plant J. 26, 89-100.

Gottwald, J. R., Krysan, P. J., Young, J. C., Evert, R. F., and Sussman, M. R. (2000). Genetic evidence for the in planta role of phloem-specific plasma membrane sucrose transporters. Proc. Natl. Acad. Sci. U.S.A. 97, 13979-13984

Hiei, Y., Ohta, S., Komari, T., and Kumashiro, T. (1994). Efficient transformation of rice (Oryza sativa L) mediated by Agrobacterium and sequence-analysis of the boundaries of the T-DNA. Plant J. 6 , 271-282.

Krapp, A., Hofmann, B., Schafer, C., and Stitt, M. (1993). Regulation of the expression of rbcS and other photosynthetic genes by carbohydrates - a mechanism for the sink regulation of photosynthesis. Plant J. 3, 817-828.

Kuroda, M., Kimizu, M., and Mikami, C. (2010). A simple set of plasmids for the production of transgenic plants. Biosci. Biotechnol. Biochem. 74, 2348-2351.

Lu, Y., Gehan, J. P., and Sharkey, T. D. (2005). Daylength and circadian effects on starch degradation and maltose metabolism. Plant Physiol. 138, 2280-2291.

McCouch, S. R., Teytelman, L., Xu, Y. B., Lobos, K. B., Clare, K.
Walton, M., et al. (2002). Development and mapping of 2240 new SSR markers for rice (Oryza sativa L.) DNA Res. 9, 199-207.

Miki, D., and Shimamoto, K. (2004). Simple RNAi vectors for stable and transient suppression of gene function in rice. Plant Cell Physiol. 45 490-495.

Miyao, A., Tanaka, K., Murata, K. Sawaki, H., Takeda, S., Abe, K., et al. (2003). Target site specificity of the Tos 17 retrotransposon shows a preference for insertion within genes and against insertion in retrotransposonrich regions of the genome. Plant Cell $15,1771-1780$

Nashilevitz, S., Melamed-Bessudo, C., Aharoni, A., Kossmann, J., Wolf, S., and Levy, A. (2009). The legwd mutant uncovers the role of starch phosphorylation in pollen development and germination in tomato. Plant J. 57, 1-13.

Niittylä, T., Messerli, G., Trevisan, M., Chen, J., Smith, A. M., and Zeeman, S. C. (2004). A previously unknown maltose transporter essential for starch degradation in leaves. Science 303 87-89.

Rösti, S., Fahy, B., and Denyer, K. (2007). A mutant of rice lacking the leaf large subunit of ADPglucose pyrophosphorylase has drastically reduced leaf starch content but grows normally. Funct. Plant Biol. 34, 480-489.

Russin, W. A., Evert, R. F., Vanderveer, P. J., Sharkey, T. D., and Briggs, S. P. (1996). Modification of a specific class of plasmodesmata and loss of sucrose export ability in the sucrose export defective 1 maize mutant. Plant Cell 8, 645-658.

Sasaki, H., Aoki, N., Sakai, H., Hara T., Uehara, N., Ishimaru, K., et al. (2005). Effect of $\mathrm{CO}_{2}$ enrichment on the translocation and partitioning of carbon at the early grain-filling stage in rice (Oryza sativa L.). Plant Prod. Sci. 8, 8-15.
Schlosser, A., Martin, J., Hannah, L. and Giroux, M. (2012). The maize leaf starch mutation agps-m1 has diminished field growth and productivity. Crop Sci. 52, 700-706.

Slewinski, T. (2012). Non-structural carbohydrate partitioning in grass stems: a target to increase yield stability, stress tolerance, and biofuel production. J. Exp. Bot. 63, 46474670.

Slewinski, T., and Braun, D. (2010). The psychedelic genes of maize redundantly promote carbohydrate export from leaves. Genetics 185, 221-232.

Slewinski, T., Ma, Y., Baker, F., Huang, S., Meeley, R., and Braun, D. (2008) Determining the role of Tie-dyed 1 in starch metabolism: epistasis analysi with a maize ADP-glucose pyrophosphorylase mutant lacking leaf starch. J. Hered. 99, 661-666.

Smith, S. M., Fulton, D. C., Chia T., Thorneycroft, D., Chapple, A., Dunstan, H., et al. (2004). Diurnal changes in the transcriptome encoding enzymes of starch metabolism provide evidence for both transcriptional and posttranscriptional regulation of starch metabolism in Arabidopsis leaves. Plant Physiol. 136, 2687-2699.

Stitt, M. (1991). Rising $\mathrm{CO}_{2}$ levels and their potential significance for carbon flow in photosynthetic cells. Plant Cell Environ. 14, 741-762.

Vriet, C., Welham, T., Brachmann, A., Pike, M., Pike, J., Perry, J., et al. (2010). A suite of Lotus japonicus starch mutants reveals both conserved and novel features of starch metabolism. Plant Physiol. 154, 643-655.

Wang, H., Qi, M., and Cutler, A. (1993). A simple method of preparing plantsamples for PCR. Nucleic Acids Res. 21, 4153-4154.

Wang, J. L., Jiang, J. D., and Oard, J. H. (2000). Structure, expression and promoter activity of two polyubiquitin genes from rice (Oryza sativa L.) Plant Sci. 156, 201-211.
Weise, S. E., Aung, K., Jarou, Z. J., Mehrshahi, P., Li, Z. R., Hardy, A. C., etal. (2012). Engineering starch accumulation by manipulation of phosphate metabolism of starch. Plant Biotechnol. J. 10, 545-554.

Yu, T. S., Kofler, H., Hausler, R. E., Hille, D., Flügge, U. I., Zeeman, S. C., et al. (2001). The Arabidopsis sex 1 mutant is defective in the $\mathrm{R} 1$ protein, a general regulator of starch degradation in plants, and not in the chloroplast hexose transporter. Plant Cell 13, 1907-1918.

Conflict of Interest Statement: The authors declare that the research was conducted in the absence of any commercial or financial relationships that could be construed as a potential conflict of interest.

Received: 05 March 2013; paper pending published: 20 March 2013; accepted: 29 April 2013; published online: 27 May 2013.

Citation: Hirose T, Aoki N, Harada Y, Okamura M, Hashida $Y$, Ohsugi $R$, Miyao A, Hirochika $H$ and Terao $T$ (2013) Disruption of a rice gene for $\alpha$ glucan water dikinase, OsGWD1, leads to hyperaccumulation of starch in leaves but exhibits limited effects on growth. Front. Plant Sci. 4:147. doi: 10.3389/ fpls.2013.00147

This article was submitted to Frontiers in Plant Physiology, a specialty of Frontiers in Plant Science.

Copyright (c) 2013 Hirose, Aoki, Harada, Okamura, Hashida, Ohsugi, Miyao, Hirochika and Terao. This is an openaccess article distributed under the terms of the Creative Commons Attribution License, which permits use, distribution and reproduction in other forums, provided the original authors and source are credited and subject to any copyright notices concerning any third-party graphics etc. 